\title{
Parler la terre : rites et rêverie dans Terre exacte (2007)
}

Ce chapitre continuera notre examen de l'œuvre tellermannienne à travers l'esprit de commémoration et de récupération - de la terre comme d'un passé collectif mythique - qui se déploie dans Terre exacte. En examinant ses rites quasi religieux et sa rêverie énigmatique, nous constaterons des liens renouvelés entre les êtres humains et le monde élémentaire, les lieux et le souvenir, la terre et le langage. Le fil conducteur sera cette fois une «terre exacte » puissante et indéfinissable, essentielle à l'être et au devenir. Comme à son habitude, Tellermann donnera voix aux disparus en se servant d'un lexique singulier et d'emprunts à Celan. Les récitatifs ainsi tissés interrogent l'être, mais dessinent aussi maints gestes vers l'Autre, insistant sur les liens identitaires et générationnels que crée le langage, ce qu'elle aime à appeler une « poussière d'étoiles retrouvée » $(N N 7)$. Sera également mise en évidence la progression de son écriture de livre en livre : chaque recueil aborde certes un nouveau topos, en l'occurrence la terre, mais en poursuivant la quête d'une parole pleinement intersubjective, de liens d'une profondeur certaine aux niveaux historique, littéraire et ontologique, ajoutant d'ailleurs aux débats qui animent la culture française depuis l'après-guerre autour du lyrisme, de l'habiter (éco)poétique et de l'acheminement vers la parole. ${ }^{1}$

Comme nous l'avons vu, l'énigme sert à explorer l'inconscient et ses signifiants. Il s'agit d'éviter la pensée conceptuelle et de mettre sur le même plan, avec douceur et générosité, le présent, le passé et le futur, notamment au moyen de longues séquences et de syntagmes énigmatiques, tel le titre de ce recueil. Façon dont Tellermann souligne les rites et la rêverie, associe le dedans et le dehors, les lieux et le souvenir, la terre et le déploiement du langage, ajoutant à la tradition poétique où l'on parle la terre en tant que moyeu d'une roue qui nous anime, point central d'où émanent notre prise de conscience du cosmos et d'autrui. Nous nous plongeons dans le paradoxe d'une «terre exacte » jaillisante, indéfinissable, souvent hors de notre portée, et pourtant au cœur de notre être-au-monde et du devenir. Tellermann évoque la terre afin

1 Cf. Martin Heidegger, Acheminement vers la parole, trad. Jean Beaufret, Wolfgang Brokmeier et François Fédier, Paris : Gallimard, 1976 [1959], ouvrage qui ne figurera pas dans nos analyses, mais qui alimente la poésie et la philosophie des dernières décennies.

(C) AARON PREVOTS, 2022 | DOI:10.1163/9789004499683_005 
de donner voix aux « dé- / nommés » (TE 246) de l'Histoire, en mots concis qui servent tant à réconcilier qu'à interroger. Les poèmes, où de brefs vers incantatoires donnent aux récitatifs tellermanniens de l'ampleur aux plans spirituel et spatiotemporel, font de nos découvertes - de la terre, du langage, du désir, de l'Autre - des rites au bord de l'indicible. Le rite et la rêverie s'intriquent, tant le vers y est allusif, paratactique, saccadé, polysémique. Les évocations d'un passé mythique suggèrent une « terre exacte » enracinée dans la légende (50), des espaces intemporels, le monde minéral et notre soif d'étendues naturelles, comme celles des Psaumes (cf. 83-132), livre sacré traité indirectement, bien qu'avec plus d'insistance qu'auparavant, pour parler plus d'un trouble généralisé - de «l'air autour de ce qui / se tait » (101) - que de croyances précises. L'analyse suivante mettra en relief la poét(h)ique de ces résonances, au regard du souvenir, du relationnel, de l'intersubjectif, de l'intertextuel et d'un dialogisme innovateur.

Le rite, ses mystères et le contact avec la création tout entière s'inscrivent d'emblée dans Terre exacte. Des vers repris d'Encre plus rouge sonnent le début d'un poème visionnaire, « inscrit sur une terre / inconcrète et sans nom » $(E R$ $4^{\mathrm{e}}$ de couv.). Nous assistons de nouveau à un drame tellurique, quasi religieux : "C'était une histoire inannoncée / face aux trois Dieux. / / Ô jour d'innocence / où nous nous avisions / et fûmes proférés / ...» $(T E 7){ }^{2}$ La présence de ces «Dieux» sans nom fait se confondre le rite et la rêverie. De tels énoncés colorent vite l'étoffe des premiers textes, si bien que le lecteur se met lui aussi à songer, à faire le lien entre les nombreuses références énigmatiques, y compris celles qui nous font explorer un passé ancestral ambigu, fluide, mi-réel, mi-mythique. Les vers isolés de cette épigraphe précédant les quatre parties «Du dit jamais» (9-82), «Psaume 9» (83-132), «Jour jaune» (133-208) et «Un nom d'homme » (209-69) présagent le lent dépliement d'un conte surgi de la légende, nous fascinent de par leur ton et leur contenu, créant une tension entre l'innocence et la détermination par une force extérieure.

Par ailleurs, la compacité, la brièveté et l'abstraction de ces vers éveillent notre curiosité, car nous ne savons pas encore quels dilemmes seront abordés, quelle histoire « inannoncée » sera traitée, à quels « Dieux » il faudra faire face et répondre, quels torts ou injustices il faudra redresser, réparer. Un jour du Jugement pourrait avoir lieu, ou bien des jours d'une plus grande innocence lorsque seront avouées les fautes. L'emploi des temps du passé crée une distanciation,

2 Cf. ER 165. Par moments, Tellermann se cite. De telles reprises renforcent la musicalité et l'urgence du dire, la découverte de soi et de la parole, le regard sur le passé et l'élan vers un avenir pleinement intersubjectif. Accueillir ainsi le langage est une façon de prier, de savourer l'énigme, de retrouver l'« innocence». 
comme le fait l'image des «trois Dieux», image qui nous situe dans le sacré tout en s'ouvrant aux confessions diverses. S'exclamer « Ô jour d'innocence », c'est invoquer un retour, plein d'émotion et de prières, à ce passé mythique. La consonance des [s] renforce les cadences des vers courts mais relativement équilibrés, proches de celles de l'alexandrin dans la deuxième strophe. Le son [e] - « inannoncée », « proférés » - équilibre les deux strophes, les ouvre au songe et au passage du souffle tout en structurant cet ensemble. La locutrice nous indique que le cour et l'esprit doivent accueillir les souffrances du passé, notamment lorsque les silences en fin de vers rendent plus vaste la portée de la pensée autour de ce dire oraculaire et abstrait, laissent au non-dit sa part. Le parallélisme des vers où figurent les mots « inannoncée » et « innocence » - « une histoire inannoncée [...] Ô jour d'innocence »- fait écho aux Psaumes. Ces vers chantonnent un hymne inattendu. Le bruissement du langage met en évidence l'émotion, la sensation, le surgissement de liens profonds.

Terre exacte baigne dans le rite et la rêverie portés par les espacements, le tissu sonore, les images, la brièveté des vers et les références faites de biais à la Bible. Plus qu'au parler quotidien, la manière dont respirent les vers, le dépouillement, les intervalles, les reprises et les unités de sens saisies par le regard posé sur la page s'apparentent aux formes liturgiques, particulièrement celles de la tradition hébraïque. Le lexique se rapportant à l'eau, à la mer, à la pierre, aux étoiles renvoie au dire à la fois élémentaire et éternel de l'Ancien Testament (cf. 13, 15). Le titre de la séquence « Psaume 9 » pointe de manière concrète ces réverbérations. L'écriture tellermannienne ressemble ainsi à ce qu'appelle Jean-Marie Perret à propos d'Encre plus rouge une « succession de petits radeaux textuels confiés à un courant qui monte en nous ». ${ }^{3}$ Il convient de noter l'aspect fragmentaire et indécis, paratactique et troué, questionnant et même balbutiant des sauts de la pensée qui serrent et ouvrent le sens. De nombreux énoncés signalent une absence, un manque, les béances ou trous sémantiques qui caractérisent tant l'extrême contemporain.

Plusieurs axes importants apparaissent dans « Du dit jamais » (9-82). L'exactitude de la terre correspond à un jaillissement intérieur, sensuel et palpable. Parler la terre vient de l'envie de commémorer, de récupérer, de se souvenir pour mieux regarder en avant. C'est tendre la main aux êtres et aux choses,

3 Jean-Marie Perret, «Esther Tellermann, Encre plus rouge », op. cit., p. 114. Cf. Celan quant aux correspondances entre les mots de la poésie et une «barque», voire sa variante plus fragile et ouverte aux éléments, un «radeau » qui traverse des courants affectifs, par lequel nous naviguons les terrains du corps ou de l'âme : « ton cœur est une barque dans le blé, nous, rameurs, la poussons vers la nuit » (Paul Celan, « Marianne», [Pavot et Mémoire], Choix de poèmes, op. cit., p. 27). 
tant du passé que du présent. C'est refondre peu à peu l'imaginaire collectif. Parler la terre exige une prise de conscience calme et sûre: non seulement nommer les parties d'un tout, mais aussi établir un monde plus harmonieux, sans oublier apories et incertitudes participant de cette appréhension socioculturelle et linguistique. Ainsi le côté élégiaque et quelque peu prophétique des premiers poèmes, où se transmet l'accueil des cycles du cosmos et des commencements qu'instaure le rêve :

Pense / aux roues lentes / au cœur d'un monde. (13)

Offre en mélancolie / la somnolence / d'un son aigre / ô du vide de toi (14)

Qui brûle les soies lissées? (15)

toi plus lourd / par où commence / / le vert du rêve. (17)

Regardant en avant et en arrière, Tellermann se rend compte non pas de scènes précises de l'Histoire, mais de fragments de sens venus de l'inconscient, de signifiants qui évoquent le pouvoir de nos gestes et sèment les graines d'un lent renouveau. Le chant brisé s'accorde aux images. Il s'agit de trouver des mots justes, sans que chaque pierre soit méticuleusement sertie (cf. 46). Emprunter à divers domaines donne mieux voix à l'indicible et au non-dit. Les « roues lentes / au cœur d'un monde», par exemple, intimement liées à divers cycles - mort et vie, jour et nuit, aubes et crépuscules qui indiquent le mouvement de corps célestes -, font peut-être allusion à la quête spirituelle de Dante, ${ }^{4}$ ainsi qu'à l'expression chez Du Bouchet des rapports entre le regard attentif et la matière qui ne cesse d'apparaître, relation qui nous fait habiter le temps, l'espace et le langage. ${ }^{5} \mathrm{La}$ « somnolence / d'un son aigre » fait écho au chant d'Orphée et à sa lyre, d'autant que la strophe parle d'un « vert troqué contre le souvenir» (14). Il y a certes de la somnolence et de la mélancolie, mais aussi

4 Dante, CEuvres complètes, trad. Christian Bec et al., Paris : LG F, 1996, Paradis XXXIII, 143-45, p. 1024 : « Mais il tournait ma soif et mon vouloir, / exacte roue, l'amour qui dans sa ronde / élance le soleil et tant d'étoiles. »

5 André du Bouchet, «Le révolu », Laisses, Paris : Hachette, 1979, n.p. (1) : « De face, comme au sol / révolu, je vois la roue de face comme rentrée, qui ramène / sans dévier à des yeux qu'on racle. » Voir aussi la lecture attentive de cette « description d'une zone inconnue » et les commentaires sur le mot « roue » dans Clément Layet, André du Bouchet : présentation et anthologie, Paris : Seghers, 2002, p. 92-114 $(97,103)$, ainsi que la discussion de la « métaphore des roues» et des «mécanismes de l'ontos» dans Michael Bishop, «Esther Tellermann: exactitude, interrogation, psaume », op. cit., p. 28. 
cette musique censée faire refleurir, enclencher un changement de saison. La question « Qui brûle les soies lissées ? » et la voix « qui seule voit / où il y a des yeux » correspondent peut-être aux vêtements, aux étoffes rituelles et aux vies perdues pendant la Shoah ou d'autres ères de destruction, et pourtant Tellermann laisse entendre que la poésie retisse les soies, ravive la parole, illumine « chaque route » par le biais de « l'étoile » qu'elle désigne, qui nomme, guide et porte espoir. De même, un poème peu après, tel un fil dans sa forme verticale, indique «ce qui ne s'assemble » mais annonce une « terre surgie dans / la voix humaine » (17). L'image du « vert du rêve » par laquelle se termine ce texte unit le tellurique et le spirituel, le mythique et l'ici et maintenant. Ces poèmes de « Du dit jamais » rassemblent les êtres et les choses qui nous sont chers. Le rite et le rêve les relient - roues, sons, soies, couleurs - au devenir dans un sens très large, de façon sensuelle et musicale autant qu'ontologique et existentielle. La profondeur des images et la fluidité paradoxale des vers incarnent la croissance du monde extérieur dont il est question, font chanter ce qui est ciselé et étroit, font résonner un dire qui est ancien de par sa concision et ses couches sémantiques, actuel de par son affrontement à l'Histoire.

Les moyens qu'emploie Tellermann pour reconnaître les disparus passent par la poétique celanienne. Lorsqu'elle s'appuie sur le rite et le rêve pour faire surgir l'autre monde, par des mots aussi compacts que ceux de Celan mais dans des séquences plus longues et berceuses, elle pense souvent à lui. Lui faire allusion l'aide à faire de l'intersubjectivité le moteur de Terre exacte, puis à proposer des liens entre le dedans et le dehors, à explorer des espaces orphiques, à renouveler la langue et l'imaginaire. ${ }^{6}$ À travers des motifs celaniens concernant les disparus, parler la terre - et le cosmos - devient plus facile. S'inspirant de lui, des tournures, des allusions et des remarques indirectes sur la souffrance humaine comme sur l'espoir créent un échafaudage pour descendre plus bas dans l'inconscient, puiser dans le rêve, le rite et le sacré. Le choix des mots souligne combien difficile peut être le dialogue sur la souffrance humaine, fragile l'état d'âme de la locutrice et des destinataires. Quant au désir de parler la terre, Tellermann communique avec et à travers Celan pour faire un pont vers l'autre monde - vers la commémoration et la consolation. En s'adressant implicitement à lui en même temps qu'aux disparus, quoique sans dire directement à qui se réfère le $\mathrm{Tu}$, elle augmente la portée de Terre exacte. Elle en augmente l'aspect intertextuel tout en contemplant sa propre inquiétude et mettant à nu

6 Cf. Jean Daive, La Condition d'infini 5 : sous la coupole, Paris : P.O.L, 1996, p. $9:$ : [L] e mot renvoie à la mémoire et [...] celui-ci est un espace imaginaire où se joue la lisibilité du monde. [...] Le monde est illisible et la matière des mots engendre une structure : le poème. La vibration du sens est utilisée comme énergie. » 
les blessures de l'Histoire. Elle fait en sorte que Celan aussi soit l'un des disparus, enrichit l'éventail de tonalités et nous rappelle comme le ferait André du Bouchet que la terre se fait plus accessible et transparente lorsque sont précisés sans cesse les liens entre la terre et chacun de nos souffles, de nos pas, de nos regards. ${ }^{7}$

Les intertextes celaniens qui sont le point d'ancrage de la rêverie dans Terre exacte permettent des lectures multiples. Ils en disent long sur l'affrontement avec l'Histoire qui y a lieu. Par exemple, les vers «Pense / aux roues lentes / au cour d'un monde» (13) citent probablement «Strette», poème écrit par Celan sur le film Nuit et Brouillard (Alain Resnais, 1955). ${ }^{8}$ "Strette» dépeint une «roue, lente», un soleil au sens figuré dont les rayons montent mais ne servent de contrepoids ni à la noirceur d'un champ ni à l'absence des morts. Le lyrisme de Tellermann en devient plus urgent, plus précis. Lu au travers de «Strette», le début de Terre exacte se fait moins abstrait, à l'égard de fils symboliques qui relient les générations, de l'étoffe de la vie en train de se former et d'une eau qui divise. Il résonne considérablement quand le fond en est «Strette», poème relativement long (pour Celan) qui pleure la Shoah, «les ravages de la bombe atomique $»^{9}$ et les peines du témoignage. L'eau dont parle Tellermann dans le poème par lequel commence « Du dit jamais », eau qui ne nous alimente pas comme elle le devrait et perturbe la perception, reprend des images de «Strette » à propos du chaos, de la désarticulation, d'un œil humide et d'yeux secs. Lire ces textes en miroir nous aide à voir, dans l'eau qui « fractionne», des yeux endeuillés et des larmes déchirantes: «Pense / aux roues lentes / au cœur d'un monde. / Qui encore porte / vos cheveux / fait tissu ? / Quelle eau fractionne / l'apparence / et devient telle / que nous l'avons vidée ? » $(T E$ 13). Une douleur semblable à celle de Celan se cristallise. Parler la terre en devient acte par lequel questionner ce qui la vide, explorer son évolution

7 Dans Une lampe dans la lumière aride, op. cit., p. 78-79, André du Bouchet montre comment dire nous aide à mieux voir et respirer. Il insiste sur le devoir de répéter sans cesse ce qui a été dit, afin de renouveler l'air que l'on respire et de se sentir chez soi (cf. Carnet, op. cit., p. 11 [avril 1951]). Chez Du Bouchet, la rencontre avec le langage ainsi qu'avec la matière du dehors comme socles sur lesquels fonder le devenir ressemble en partie à celle qui caractérise l'œuvre de Celan. À propos du souffle - de la respiration - comme élément primordial du devenir, voir aussi l'emploi du mot « habitacle» dans André du Bouchet, Carnet 2, Saint Clément: Fata Morgana, 1998, p. 100: «au centre de l'air / une / figure en nous / dans son habitacle transparent / / partout le centre, brèche dans le pourtour »; cf. GE 147 : « nos rives seraient bordées / nos habitacles / et l'ampleur de la mer ».

8 Paul Celan, Choix de poèmes, op. cit., p. 154-69, p. 345n1; cf. « Le banquet », p. 32-35. L'image d'une « roue, lente » apparaît dès le début du poème « Strette », p. 154-55.

9 Paul Celan et Erich Einhorn, « Correspondance Paul Celan / Erich Einhorn - Extraits », lettre du 10 août 1962, Paul Celan, Europe 861-862 (janv.-févr. 20o1), p. 56-62 (58). 
et ses saisons, et se faire porte-parole des morts et des vivants. Des stratégies bien tellermanniennes - le Tu implicite de l'impératif, le Vous derrière l'énoncé « vos cheveux », le Nous de la deuxième question - rassemblent toutes sortes de destinataires : le poète, d'autres écrivains, des bien-aimés, le lecteur, la lectrice, ceux et celles qui nous ont précédés et qui enrichiraient nos réflexions sur l'élémentaire et le tellurique, le cosmos et le cheminement humain. En outre, ces vers nous font voir Terre exacte en tant que strette, mot clé du lexique celanien : la partie d'une fugue où, avant que n'arrive la conclusion, les entrées du thème se multiplient et se chevauchent, nous rendent plus attentifs à la façon dont elles se complètent, à la musicalité elle-même, dans l'évocation du souvenir et de l'oubli, de la commémoration et la consolation, du dialogue et de la guérison. ${ }^{10}$

Quant au poème en tant qu'acte qui crée des liens entre la croissance humaine et l'élémentaire, «Strette » suggère que l'eau des profondeurs de la terre s'inscrit devant nous par le biais de dialogues que tracent métaphoriquement devant les yeux du locuteur une chouette et l'herbe qui couvre des pierres tombales. En allemand, les derniers vers de «Strette» enveloppe ce chant funèbre de musicalité hésitante, de sons répétés que tente de reproduire la traduction à travers les lettres $\mathrm{h}$, e et $\mathrm{t}$ : « Herbe. Herbe, écriture désarticulée ».11 Tellermann ouvre «Du dit jamais » avec une musicalité semblable à celle de Celan mais qui vise d'autres effets. Il s'agit, dans la mesure du possible, d'édifier, de rassurer. « Peut-être la mer est déjà / en dessous / entre les cordes » (13): ces derniers vers examinent des liens entre le dehors et notre cheminement affectif, sociétal, spirituel, ontologique. Leur progression suggère le mouvement et l'émotion au moyen du son [d], du flux et du reflux implicite de la mer et du mot « cordes », lequel correspond ici aux roues lentes qui font tourner en douceur le monde, ainsi qu'au corps, à la corde sensible sur laquelle joue le poème et à la musique elle-même. Anticipant sur d'autres allusions - « une couronne d'air » (16), « l'autre source » (19), une « rétine ouverte » $(25)^{12}-$, ce texte prolonge le dire celanien, mais établit une vision plus fluide, ouverte, généreuse. Il reprend le paradigme - si cher à Celan - de la parole fondatrice, et situe l'espoir dans les distances que crée, mesure et médite cette parole, entre soi, le monde

\footnotetext{
10 «Strette» termine le receuil Grille de parole (1959). Voir Paul Celan, Choix de poèmes, op. cit., p. 154-69.

$11 \quad$ Ibid., p. 169.

12 Des images similaires se trouvent dans Paul Celan, Strette \& autres poèmes, trad. Jean Daive, Paris : Mercure de France, 199o. Voir p. 15 (« Nuit», [Grille de parole]), p. 53 (« Chymique », [La Rose de Personne]) et p. 81 («Dans le chariot à serpents», [Renverse du souffle]).
} 
et le langage. ${ }^{13}$ Il parle la terre, mais embrasse le relationnel et le simultané, bien au-delà du concept. Il commence un dialogue maintes fois poursuivi, au sein des séquences de Terre exacte et comme reflet illuminant du texte liminaire. Les thèmes implicites en sont nombreux : l'innocence et la perte de l'innocence ; la grâce retrouvée ; le devoir depuis la Shoah de refaire le tissu social et l'ordre symbolique ; le renouveau observable dans le monde extérieur et que peut mettre en branle le poème ; et l'aspect sacré du dire devenant cadre dans lequel nous pleurons les morts, leur parlons et relançons leur parole. Comme c'est le cas chez Celan, la rêverie en devient ainsi décidée, intentionnelle, acte auquel revenir aussi souvent que possible. Elle sert à constater la valeur de la vie humaine et à relier celle-ci au temps, à l'espace, à la nature, aux cycles qui nous entourent et animent.

Le ton cérémoniel de Terre exacte s'éploie peu à peu. Les poèmes sans titre se suivent, intégrant mais transformant en même temps des aspects de Celan. À travers un élan particulièrement intersubjectif, des motifs récurrents et des séquences très amples qui développent avec intensité les interrogations, Tellermann fait s'entrelacer des fils: le passé et le présent, la terre et ceux qui l'habitent, la répétition de rites et le silence ou le chant qui les accompagne. Quoiqu'en se limitant, au niveau du cadre narratif, à quelques éléments de base tels que la rêverie, le respect pour les disparus et l'acheminement vers la contemplation, elle met en avant des actions ayant un sens symbolique. Les énoncés nomment les distances entre le présent et le passé, mais les réduisent tout autant. Nous sommes aux prises avec l'absence de ceux qui nous semblent présents, cette « voix de personne » $(15,24)$ qui, au fil des images, est le « rien » affirmant une absence dans la voix de la locutrice (15), s'avère « cousue » sous sa peau (24), met la détresse «entre nos mains » (24) et cristallise le «rien » ressenti « entre les dents » et « sous la paupière » $(25) \cdot{ }^{14}$

Dans Terre exacte, la couleur met en évidence ce paradoxe de la présence-absence des êtres et des choses, leur proximité et leur distance dans le temps et l'espace, notre relation à leur visibilité et à leurs gestes expressifs. Puisque Tellermann a pour ainsi dire un coup de pinceau large et non-représentatif, mettant de côté le portrait et favorisant les grands traits que mettent en place le signe, la couleur joue un rôle important dans ce recueil. Celle-ci communique

13 Cf. Paul Celan, Le Méridien \& autres proses, op. cit.; Frédéric Marteau, « Contra-diction. Paul Celan et l'art du contrepoint », Littérature 180 (2015/4), p. 56-69; et Jean-Michel Maulpoix, Jean-Michel Maulpoix commente Choix de poèmes de Paul Celan, Paris : Gallimard, 2009.

14 Vis-à-vis d'une «voix de personne» et de l'innommable, voir Paul Celan, «Un œil ouvert », [Grille de parole], Strette \& autres poèmes, op.cit., p. 19. 
ce qui a lieu aux niveaux de l'affect, de la pensée et de la perception lorsque nous nous souvenons de traumas divers ayant des conséquences sur le réel. De même que Tellermann reste laconique sur les détails d'un paysage, elle se sert de mots liés à la couleur pour mettre l'accent sur un certain nombre de champs sémantiques, nous faire (re)connaitre intuitivement la pertinence de l'image. Utiliser la couleur ici renvoie à l'idée que la terre n'est « exacte » que lorsque l'imaginaire en détermine les contours. Il s'agit d'accéder fugitivement à des strates spatiotemporelles qui s'étendent jusqu'à l'Antiquité. Son parti pris esthétique et ontologique - son désir d'aborder à travers la couleur la vie, la mort, l'immensité de la terre, l'illimité des liens humains - ressemble par exemple à celui de Hölderlin dans « En bleu adorable », 15 ou de Hugo dévoué à la moindre nuance de « l'éternel murmure ». ${ }^{16}$ Or la couleur, comme chez Trakl dont les textes sombres «tir[ent]» souvent «sur le bleu » ${ }^{17}$ ou comme dans Encre plus rouge où le rouge se lie autant à l'affect qu'à l'élémentaire, garde son élan énigmatique, son caractère insistant. Notons, lorsque la pensée s'engage dans la couleur dans Terre exacte, les émotions et sensations qui viennent en partage. $^{18}$

La couleur s'avère fondamentale quant aux rites et rêveries de Terre exacte. À la manière d'Encre plus rouge, où le rouge informe les séquences « Rouge couleur des nerfs » et «Encre plus rouge», la séquence «Jour jaune» dans Terre exacte (133-208) privilégie le jaune. Avant tout, il est question de l'exploration cérémonielle du temps et de l'espace, de la vie et de la mort, du langage et des relations humaines. L'auteur ne souligne pas le sens précis, systématique, symbolique d'une couleur. Plutôt, elle montre comment la couleur, le dire, les éléments et le signe s'entrecroisent. «Jour jaune » met l'accent sur des « liturgies nues » (148) qui créent des zones d'ombre et de lumière et se mettent à inonder le signe $(137,140)$. Leur bruissement (cf. 38, 87, 177, 263) ranime la présence des êtres et des choses, comme dans les vers «Donne les jaunes / mendiés » (146) et «Jour jaune / sur eux » (150), où s'annoncent des «alliances » (169) entre les morts et les vivants, configurées de manière relativement vive

15 Celan s'appuie sur ces mêmes idées directrices et s'intéresse également à la poésie de Hölderlin. Voir Friedrich Hölderlin, «En bleu adorable », [trad. André du Bouchet], CEuvres, op. cit., p. 937-41.

Victor Hugo, «Ce que dit la bouche d'ombre », Les Contemplations, éd. Pierre Laforgue, Paris : Flammarion, 20o8, p. 361-83 (362). Georg Trakl, « À un jeune mort », Vingt poèmes, trad. et préface Eugène Guillevic, Sens : Obsidiane, 2016, p. 45-47 (45).

18 À propos de liens entre la couleur et l'écriture poétique, voir aussi Paul Celan, «L'écrit », [Renverse du souffle], Strette \& autres poèmes, op.cit., p. 93 : « L'écrit se creuse, le / dit, vertocéan, / brûle dans les baies. » 
au moyen de consonances et de gestes indiquant la générosité humaine et le passage du temps. Au début de «Jour jaune », cette couleur correspond plus ou moins à la clarté émergeant du langage (137), à l'or liquide que contient un nom (138) et à d'éventuels reflets des « lampes » mettant en lumière des liens interpersonnels, des «mains cousues» (140). Le jaune s'assimile également, de façon plus familière, aux fleurs et aux paysages : « un bouquet / jaune » (233); « des cités / jaunes » (269). Dans l'ensemble, il incarne le topos de la lumière qui nous nourrit, qui traverse le langage et les émotions, tels les «psaumes / répétés » qui sont un « grand soleil sorti de nous / / dédié au sacrifice » (201). ${ }^{19}$ Comme le bleu, le rouge, le vert, le noir et le blanc dans Terre exacte, c'est une couleur qui fait coïncider la matière et l'esprit. Le jaune nous met presque en osmose avec le monde extérieur et ses parcelles, comme une interlocutrice serait « enroulée dans le jaune » (44). Pour toutes ces raisons, nous comprenons que la poésie réduit les distances entre les êtres et les choses, transforme ces distances en promesse, relie terre et ciel, qu'il s'agisse d'« horizons de sel » à proximité (141) ou de « toute pierre qui s'ouvre / pour la faille brillée / / du milieu » tout près (44).

Considérons aussi le jade, couleur et pierre bleu-vert en symbiose avec le rite, la terre et les croyances de l'Antiquité. Par le biais du jade, dont le nom vient de ce que l'on pensait être ses pouvoirs curatifs, ${ }^{20}$ le rite et la rêverie font encore plus partie d'un processus de guérison. Souvenons-nous des vers cités plus haut concernant « un vert troqué contre le souvenir » (14) et «le vert du rêve » (17), lesquels annoncent plus généralement l'importance de la couleur en tant que fil tout au long de Terre exacte, puis renvoient aussi au titre, à savoir l'idée que seront mis en valeur les lieux et l'espace, la mémoire et l'oubli, l'exploration et la croissance, la terre et ses capacités régénératrices. Peu après, le jade entre en jeu pour rappeler des rites funéraires, mais sert aussi à mettre en relief un respect cérémoniel pour la terre et ceux qui l'habitent, au-delà de gestes isolés. C'est même une sorte de mantra dans « Du dit jamais » : il faut mettre le jade « dans la bouche » $(27,30,32,67 ; \mathrm{cf} .175,176)$, motif qui évoque des rites anciens et renforce la présence de cérémonies dans ce récit-récitatif. Le contact

19 Cf. Paul Celan, «Soleils-filaments », [Renverse du souffle], Strette \& autres poèmes, op.cit., p. 79, où s'entremêlent la pensée, la lumière, les vers du poème, la présence d'un arbre et des chants à chanter «au-delà des hommes ». De même, il faudrait noter à la fin du poème «L'écrit », [Renverse du souffle], p. 93, les correspondances entre la couleur, la lumière, des présences humaines et le surgissement des vers : «[Q]ui / dans ce / carré d'ombre / suffoque, qui / sous lui / en lueur émerge, émerge, émerge ? »

20 Le New Oxford American Dictionary, Cupertino: Apple, 2015, donne l'étymologie suivante : «Fin du XVI ${ }^{\mathrm{e}}$ siècle, du français le jade (autrefois l'ejade), de l'espagnol piedra de ijada "pierre du flanc" (pour les coliques, qu'elle était censée guérir)» (nous traduisons). 
avec le jade est revigorant en ce sens que sa matière semble répandre de la lumière et de l'énergie vitale, y compris de ses «fines nervures diversement colorées ». ${ }^{21}$ Dans les vers « Donne / lunes nuits vertes / / mets le jade dans la bouche » (32), c'est comme si ces qualités élémentaires se faisaient absorber par le corps humain - comme si de nombreuses lunes et «nuits vertes », mises symboliquement dans la bouche des disparus, assuraient que ceux-ci restent liés à la terre et au ciel, en accord parfait avec le cosmos et sa vitalité.

Le jade se rapporte à divers sens symboliques dans Terre exacte, en particulier le paradoxe d'une «terre exacte» qui résulte d'une vision ancrée non pas dans un lieu précis, mais dans le sacré et le rêve. Cette pierre précieuse reflète l'intérêt que porte l'auteur aux pratiques d'antan. En effet, le jade incarnait souvent des vertus venues d'en haut et, en Chine et au Mexique, préservait les morts de la décomposition. ${ }^{22}$ Terre exacte nous montre donc comment se nourrir spirituellement, se maintenir en vie à travers la matière, et ne donne pas d'indications sur l'enracinement dans des lieux de notre ère. À cet égard, les vers suivants sont révélateurs : « Mets le jade dans la bouche / sur les tempes / les tentures / bleu suspendu aux racines » (30). Comme chez Celan, l'image de la matière mise en contact avec la bouche connote le dire poétique. ${ }^{23} \mathrm{En}$ rapprochant le jade des rites et des couleurs, la locutrice s'exhorte à exprimer le deuil et à relier des royaumes. Le jade sert non seulement d'amulette, mais aussi d'emblème du caractère inséparable de la terre et du ciel. Alors que chez Celan les défunts semblent monter dans le ciel et y retrouver péniblement des racines, ${ }^{24}$ chez Tellermann un dire aussi compact - tel le bleu qui connote la pierre autant que le ciel - nous fait traverser de vastes étendues afin de nous les faire habiter ensemble, en nous défaisant au fur et à mesure du chagrin. Le jade met en lumière cette sensibilité à l'espace, au temps, à l'Autre, aux réalités disparates.

$21 \quad$ Michel Cazenave, éd., Encyclopédie des symboles, op. cit., p. 328.

22 Ibid.; J Jean Chevalier et Alain Gheerbrant, éd., Dictionnaire des symboles, op. cit., p. 52728 ; Nanon Gardin et al., Petit Larousse des symboles, op. cit., p. 351.

23 «Quelque chose pousse dans la bouche» (TE 70). Celan, de même, met en évidence la possibilité pour les défunts de revenir de leur exil grâce aux « gerbes d'espoir » que laisse pousser le poème et qui, éventuellement, effaceraient le choc visible dans leurs « bouches éblouies ». Voir « En l'air là-haut... », [La Rose de Personne], Choix de poèmes, op. cit., p. 223.

24 Celan suit la tradition kabbaliste en pensant aux racines qui poussent dans le ciel, là où se trouve l'arbre de la kabbale qui grandit de haut en bas, du firmament vers l'humanité. Voir « En l'air là-haut... », [La Rose de Personne], Choix de poèmes, op. cit., p. 220-23 et p. 221n1. D'autres renversements paraissent souvent chez Celan, par exemple lorsque les étoiles symbolisent ceux et celles qui habitent notre cœur et qui sont aussi des fleurs. Voir « Une étoile de bois », [Grille de parole], et « Odeurs d'automne muette », [La Rose de Personne], Strette \& autres poèmes, op.cit., p. 21 et p. 47. 
Une autre page où résonne le refrain «mets le jade dans la bouche » (32) est elle aussi gnomique, oraculaire, ambivalente, et pourtant encourageante :

\author{
Ici n’a pas encore \\ l'usure des déserts \\ ici nous remontons les boues \\ ô hommes pourrissants \\ sachez encore \\ les arceaux \\ âme avide de réséda \\ d'ocre rouge \\ de chaux d'eaux rases. \\ Nous avons séché le sang
}

mis le jade dans la bouche. $(67)$

Ce texte parle la terre pour nous faire penser à un processus, aux étendues spatiotemporelles par lesquelles nous passons dans la vie ou dans l'imaginaire. Il traite surtout de la matière avec laquelle nous entrons sans cesse en contact. C'est comme si le Nous risquait de créer un monde sauvage inadéquat, et comptait sur le rite pour maintenir ses valeurs. La couleur qui s'insinue dans ce poème indique combien nous habitons la terre, aux plans social, psychologique, spirituel, ontologique. Le ricochet des sons - [d], [bu], [s], [z] - fait avancer le récit, « remont[er] les boues », désirer les choses de la terre tel le jade ou le réséda. ${ }^{25}$ Le lexique, les couleurs et la compacité de ce poème s'harmonisent avec l'exploration ambitieuse dans Terre exacte des « arceaux » du monde, des « cambrure[s] » (241) du dedans et du dehors, des écueils du dire qu'il faudrait contourner. Le courbé correspond au rite et à la rêverie, aux gestes cérémoniels ainsi qu'aux chemins indirects que suit la pensée, à la réalité pleinement présente qui pourtant se dérobe. Il est question d'établir un tout cohérent en cousant des fils où se complètent les parties de ce tout, par exemple les déserts et la civilisation, la vie vigoureuse des plantes et celle de l'âme, la terre et les eaux, le jade et les défunts qui attendent la commémoration. Les derniers vers de ce poème mettent en valeur un tel langage symbolique, par lequel les êtres et les choses façonnent les lieux qui nous entourent et nous habitent.

25 Quant à la polysémie, le réséda correspond soit à une couleur - le vert pâle -, soit à une plante. Le mot réséda évoquait à l'origine les qualités médicinales de celle-ci : « Milieu du XVIII ${ }^{e}$ siècle, du Latin, à partir de l'impératif de resedare et du champ sémantique "soulager" » (New Oxford American Dictionary, op. cit.; nous traduisons). 
C'est en parlant de la terre que Tellermann nous amène vers un avenir partagé. Lénigme sert de maillon dans cette chaîne symbolique. La prière peut en faire partie indirectement, par exemple là où il convient d'écouter une « ode » pour reconnaître par le chant « ce qui n'est pas encore » (217), pour atteindre la rive de l'Autre en présence des « mains » (26o) qui écrivent. Le rite et la rêverie vont de pair avec le désir de renouveau, créent un accord avec chaque « journée humaine» qui «s'exhale» des poumons (241). La terre en tant que cadre du dire poétique nous aide à saisir de tels parallèles entre le va-et-vient du dehors et celui du dedans, surtout à propos des changements élémentaires qui ont toujours lieu mais qui ne se voient qu'avec le temps. Lieu de métamorphoses en haut et en bas, ainsi que d'évolutions au niveau tectonique, la terre «saillie » (112) ou «emplie d'une / première peau » (119) peut devenir vertigineuse (112) ou diminuer «dans le songe » (154). La rêverie permet cette respiration de l'élémentaire. C'est comme si l'auteur glanait dans des roches autant que dans des champs, dans l'inconscient autant que dans la nature, par exemple là où des désirs sont «moissonnés » (87), où il peut y avoir des «branchages en fusion » (119). Le temps, comme la terre, doit rester indéterminé plutôt que « ce qu'on mesure », afin que chaque parcelle de réalité - chaque mot - reste une « offrande » venue en partie de la «bauxite» si riche en sédiments et donc riche en « mémoires » $(69) .{ }^{26}$

La poétique celanienne est de nouveau une grille de lecture indispensable. Nous gardons tel Orphée le cœur et l'esprit, les yeux et les oreilles, ouverts au chant des « dé- / nommés » (246) qui sont morts trop tôt ou sans deuil. C'est comme si les disparus étaient tout de même là parmi nous, couchés dans la terre ou le ciel, attendant que la parole leur soit adressée, nous amenant vers «le bord / / du dit» (30). La terre rappelle le chant qu'écoutent les vivants et les morts, ceux-ci en tant que présences qui vont à la dérive vers les parois perméables entre cette vie et «l'autre monde $» .{ }^{27}$ Plusieurs textes dans Terre exacte creusent le paradigme celanien du mot capable d'unir soi, le monde et

26 Vis-à-vis des correspondances entre le dehors et le dire poétique qui se veut commémoratif, voir les vers suivants de Celan pour leurs images et leurs parallèles avec l'écriture tellermannienne : « l'écriture de faucille a sans faire de bruit percé [...] - Lèvre privée du pouvoir de parole, fais savoir / qu'il se passe toujours, encore, quelque chose, / non loin de toi » («Reste chantable », [Renverse du souffle], Choix de poèmes, op. cit., p. 243) ; « une / drague / à contretemps passe à travers nous, ennuagés » (« Non lavés, non maquillés », [Partie de neige], Strette \& autres poèmes, op.cit., p. 121) ; « depuis les temps du rêve / perdu [...] le chœur / des souches de platanes / se voûte pour la prière / contre la prière » ( « Or », [Enclos du temps], Strette \& autres poèmes, op.cit., p. 127).

27 L'allusion est à Paul Celan, « Non lavés, non maquillés », [Partie de neige], Strette \& autres poèmes, op.cit., p. 121. 
l'Autre, par exemple le «mot lesté » dans lequel «trouver l'étoile» (153). Dans un poème qui pose la question, «Quoi atteste / la terre ? » (70), il y a les vers « toi / matière non pétrie / tresses en bordure / des quartz » (70; cf. 37), réponse soit qui fait de la terre la preuve de toutes sortes de présences humaines, soit qui fait du destinataire une entité absente. ${ }^{28}$

Plus Terre exacte avance, plus se précisent les allusions à Celan. Les énoncés mettent en évidence ses hymnes heurtés et pourtant tenaces à des bien-aimés devenus cendre ou neige, des souvenirs insaisissables mais jamais oubliés. Celan redevient l'intermédiaire qui permet à Tellermann de s'adresser en douceur à eux - «nous cherchons dans les lampes / nos mains cousues » (140); « De ta langue-fossile / je cherchais la muraille [...] Je t’ai porté afin que tu me portes » $(179) ;$ « je plante dans ta langue / le drapeau à prières » (189); « Aux psaumes / répétés vôtres / avancées de briques et d'âmes » (201) - et, en même temps, celui à qui les chants de Terre exacte s'adressent. ${ }^{29}$ La terre se fait lieu de l'affect et de l'ontos, où il est possible de porter l'Autre, autant que cadre dans lequel le signe peut recommencer à porter sens.

En revenant en arrière, au texte liminaire de Terre exacte, nous remarquons mieux la progression du recueil, les dilemmes confrontés et les désirs abordés, de façon envoûtante « où chair prit chair » $(238)$ ou en basculant « vers le

28 Celan évoque parfois le fait de pétrir ou de façonner en tant quacte poétique, biblique, mystique. L'exemple le plus connu de cet acte est peut-être le Golem, figure légendaire de la tradition hassidique en Europe centrale. Pour Celan, le mot pétrir renvoie à l'idée que Dieu, la terre et le langage nous donnent la vie. Voir « Psaume » et « En l'air là-haut », [ $L a$ Rose de Personne], Choix de poèmes, op. cit., p. 181 et p. 221 : « Personne ne nous pétrira de nouveau de terre et d'argile, / personne ne soufflera la parole sur notre poussière. / Personne »; « En l'air là-haut, c'est là que demeure ta racine, là, / en l'air. / Là où le terrestre se met en boule, terreux, souffle-et-glaise ». Voir aussi « Sous la peau », [Renverse du souffle], Strette \& autres poèmes, op.cit., p. 87 : «Quand je pétris le grumeau d'air, / notre nourriture ».

29 Vis-à-vis de mains, voir par exemple Paul Celan, «Sous la peau », [Renverse du souffle], Strette \& autres poèmes, op.cit., p. 87 : «Sous la peau de mes mains cousu: / ton nom consolé / avec des mains. » Cf. « Par le grand... », [Renverse du souffle], Choix de poèmes, op. cit., p. 241, p. 364nı et p. 364n3. Ce dernier poème se prête à plusieurs lectures. En donnant libre cours à l'imagination, on peut penser en lisant les vers suivants de ce poème soit à l'Esther biblique, soit à la Shekinah, présence divine en l'absence de Dieu parfois associée à Esther et qui selon certains aurait perdu les yeux à force de pleurer, soit à Tellermann elle-même : « Une main d'aveugle, elle aussi dure comme étoile / d'avoir traversé les noms, / repose [...] sur toi, / Esther.» Quant au «drapeau à prières » (TE 189), voir «Avec des mâts contre la terre chantés», [Renverse du souffle], Strette \& autres poèmes, p. 75 : «Tu es à l'épreuve du chant / l'oriflamme. » Autres références : «Schibboleth », [De seuil en seuil], Choix de poèmes, p. 113 : « le drapeau auquel je n'ai / prêté aucune espèce de serment »; «Strette», [Grille de parole], p. 165 : « et / le monde, mille-cristal / a déferlé ». 
vertige » (112). Nous constatons que le rite est une voie vers la guérison et que le songe est une façon d'explorer le réel. Le dire indirect de Tellermann oblige chaque rêveur à assumer la responsabilité de la langue, ${ }^{30}$ à confronter le « jour d'innocence » (7) où s'affirment par la parole des rapports à soi, à l'Histoire, au sacré, au monde naturel. Chaque lecteur est engagé dans un devenir collectif. Tous les lieux, dans l'« âme avide » (67) ou « en vue des cités / jaunes » (269), portent à travers le chant brisé la marque de ce devenir, de l'identité en train de se former. Le défi relevé est celui de penser autrement, généreusement, en prenant en compte l'intersubjectif, leçon apprise auprès des parcelles de la matière que sont le signe linguistique, la lumière et la couleur, les couches géologiques. Renvoyant aux «trois Dieux » (7) du début, la religion est un phare perçu dans le lointain, nous illuminant implicitement, momentanément, au travers de motifs symboliques, de gestes et de présences sacrés.

Un pilier de ce paradigme poétique est le dialogue. Parler la terre chez Tellermann, c'est renouer avec la langue, le cosmos et autrui. C'est se plonger dans l'apparaître et les « cycles » (200), noter d'infimes changements dans le dehors autant que dans la lumière que nous apporte le poème. Il importe dans Terre exacte d'intégrer au dire les défunts, de faire en sorte que la langue se souvienne d'eux, qu'ils se sentent écoutés d'une part, et, de l'autre, continuent grâce à l'ordre symbolique à faire partie de notre compréhension du temps, de l'espace et du monde extérieur. Tellermann regarde de près ces rapports. Le relationnel, considéré à travers la terre, devient indissociable de l'identité et de l'être-au-monde. La littérature retisse ainsi des liens entre les générations, particulièrement en ce qui concerne la commémoration de la Shoah, comme on le verra dans le chapitre suivant, et sans oublier le dialogue avec soi qu'est aussi la découverte d'un « paysage intérieur » (4 ${ }^{\mathrm{e}}$ de couv.). Parler la terre, faire appel aux disparus, entamer le dialogue : ces actes mettent en avant des enjeux civilisationnels et écopoétiques, tout en résistant au concept et en inscrivant dans la parole un «non-savoir » ${ }^{31}$ pour que l'on tienne mieux debout. De par ses énigmes et son ampleur, Terre exacte propose au lecteur de faire de tels gestes vers l'Autre : de goûter au «sel» de la parole (269) et ainsi de faire face aux échecs de l'humanité, de recueillir le sacré sous les mots.

30 Cf. Jacques Derrida, «La langue n'appartient pas : entretien avec Evelyne Grossman », Paul Celan, Europe 861-862 (janv.-févr. 2001), p. 81-91 (91): «[L]e poète est quelqu'un qui a affaire en permanence à une langue qui se meurt et qu'il ressuscite [...] Le poète [...] a la responsabilité, une très grave responsabilité, de la réveiller, de la ressusciter [...] comme un corps mortel, fragile, quelquefois indéchiffrable comme l'est chaque poème de Celan. »

Esther Tellermann, « Cristal exact », op. cit., p. 43 ; cf. TE 58, 116. 